\title{
Pattern of antigen expression in metastases after radioimmunotherapy of a syngeneic rat colon carcinoma utilizing the BR96 antibody
}

\author{
Erika Elgström ${ }^{1 *}$, Sophie E Eriksson ${ }^{1}$, Tomas G Ohlsson², Jan Tennvall ${ }^{1,3}$ and Rune Nilsson ${ }^{1}$
}

\begin{abstract}
Background: Repeated administration of antibody-based therapies such as radioimmunotherapy depends on preserved antigen expression in tumor lesions. The purpose of this study was to evaluate whether the antigen expression in metastases observed after radioimmunotherapy differs from that of untreated primary tumors.

Findings: 30 of the 35 Brown Norway rats with syngeneic colon carcinoma treated with $400 \mathrm{MBq} / \mathrm{kg}$ ${ }^{177}$ Lu-DOTA-BR96 exhibited consistent complete response of the primary tumor. 13 animals developed metastases that were detected after treatment. The antigen expression was reduced in 17 of 23 metastases detected after radioimmunotherapy compared with untreated tumors. No tumors completely lacked positively stained tumor cells.

Conclusions: Although it was not possible to demonstrate that the antigen reduction is triggered by the radioimmunotherapy this result stress the importance of considering the risk of reduced antigen expression in metastases after radioimmunotherapy prior to further targeted therapies.
\end{abstract}

Keywords: Radioimmunotherapy, Rat colon carcinoma, ${ }^{177} \mathrm{Lu}$, Antigen expression, Metastasis

\section{Background}

Treatment of primary tumors is often successful compared to that of metastatic disease, which is responsible for $90 \%$ of cancer mortality [1]. Radioimmunotherapy, the use of radiolabeled antibodies to localize radiation to the tumor, is considered a suitable treatment modality for smaller metastases [2]. Repeated administrations of radioimmunoconjugate may be required and/or beneficial in order to reduce toxicity and improve the outcome [3].

The aim of the present study was to compare the antigen expression and distribution in metastases, detected after consistent complete response (CR) of the primary tumor following radioimmunotherapy with ${ }^{177} \mathrm{Lu}$-DOTABR96, to that of untreated primary tumors in a syngeneic immunocompetent rat colon carcinoma model.

\footnotetext{
* Correspondence: Erika.elgstrom@med.lu.se

${ }^{\dagger}$ Equal contributors

'Department of Oncology, Lund University, Lund, Sweden

Full list of author information is available at the end of the article
}

\section{Findings}

Radioimmunoconjugate and tumor model

BR96 is a monoclonal antibody which binds to the Lewis $\mathrm{Y}$ antigen $\left(\mathrm{Le}^{\mathrm{y}}\right)$. Conjugation with the DOTA-chelate (S-2-(4-isothiocyanatobenzyl)-1,4,7,10-tetraazacyclododecane tetraacetic acid; Macrocyclics, Dallas, TX) and subsequent radiolabeling with ${ }^{177} \mathrm{LuCl}_{3}$ solution (MDS Nordion, Vancouver, Canada) was performed according to Eriksson et al. [4]. The radiochemical purity of ${ }^{177} \mathrm{Lu}-\mathrm{DOTA}-\mathrm{BR} 96$ was analyzed using ITLC and found to be $>97.5 \%$. Less than $1.3 \%$ labeled aggregated fractions was detected with HPLC.

Brown Norway (BN) rats were used (Harlan, Horst, Netherlands). Animals of this rat strain are immunocompetent and express the BR96 binding antigen (Lewis Y) in some normal tissues, mainly in the epithelium of the gastrointestinal tract [5], and therefore mimic the clinical situation.

The animals were inoculated under anesthesia (Isoflurane, Abbott Scandinavia AB, Solna, Sweden) with $3 \mathrm{x}$ $10^{5}$ BN7005-H1D2 cells (a cell line established from a 1,2-dimethylhydrazine-induced colon carcinoma in a BN

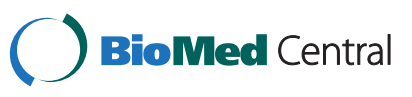


rat), between the peritoneum and the abdominal wall. The resulting local tumors were designated primary tumors. Tumor volumes were calculated as tumor length $\mathrm{x}$ tumor width ${ }^{2} \mathrm{x}$ 0.4. All experiments were conducted in compliance with Swedish legislation on animal rights and protection, and were approved by the Regional Animal Ethics Committee. The animals were housed under standard conditions and fed with standard pellets and fresh water, ad libitum.

\section{Radioimmunotherapy with ${ }^{177}$ Lu-DOTA-BR96}

Thirty-five male rats were treated with $400 \mathrm{MBq} / \mathrm{kg}$ ${ }^{177} \mathrm{Lu}$-DOTA-BR96 (150 $\mu \mathrm{g}$ DOTA-BR96 in $0.4 \mathrm{ml}$ saline with $1 \%$ human serum albumin) by intravenous injection 13-14 days after cell inoculation Group A). 11 tumorbearing rats were not treated (Group B). The treated animals were monitored for up to 120 days after injection of ${ }^{177} \mathrm{Lu}$-DOTA-BR96. The control animals (Group B) were euthanized and dissected on the day of administration of radioimmunotherapy to Group A (day 0). The median primary tumor volume on the day of treatment was $490 \mathrm{~mm}^{3}$ (range $115-1490 \mathrm{~mm}^{3}$ ) and the mean body weight was $253 \mathrm{~g}$ (SD $23 \mathrm{~g}$ ).

Twice a week, body weight of the animals in Group A was recorded, and the diameters of the primary tumors were measured with a digital caliper under anesthesia Consistent CR was defined as a non-palpable tumor during the rest of the observation period. Rats were sacrificed if the dimensions of the tumor exceeded $25 \times 25 \mathrm{~mm}$, or at the end of the study (120 days). If body weight decreased by more than $20 \%$ compared to normal weight progression, or if the general health of the animal was affected during the study, metastatic disease was suspected and the rat was sacrificed and dissected to confirm metastatic growth. At the time of sacrifice, all rats were dissected systematically by the same person, and the location and number of metastatic sites was registered. Tumor findings were fixed in $4 \%$ paraformaldehyde and embedded in paraffin.

Of the 35 male $\mathrm{BN}$ rats treated with $400 \mathrm{MBq} / \mathrm{kg}$ ${ }^{177}$ Lu-DOTA-BR96 (Group A) 30 exhibited consistent $\mathrm{CR}$ of the primary tumor. Eleven of these 30 rats developed metastatic disease and metastases were detected in another 2 rats at autopsy at the end of the study. The time at which metastatic disease was observed, and the location of metastases in various organs are presented in Figures 1 and 2. In the untreated group (Group B), all 11 animals were dissected on day 0 . None of these animals had visible metastases at autopsy.

\section{Antigen expression and distribution in tumors}

Paraffin embedded tumors and metastases were sectioned and stained with hematoxylin and eosin, and by immunohistochemical staining for the detection of the

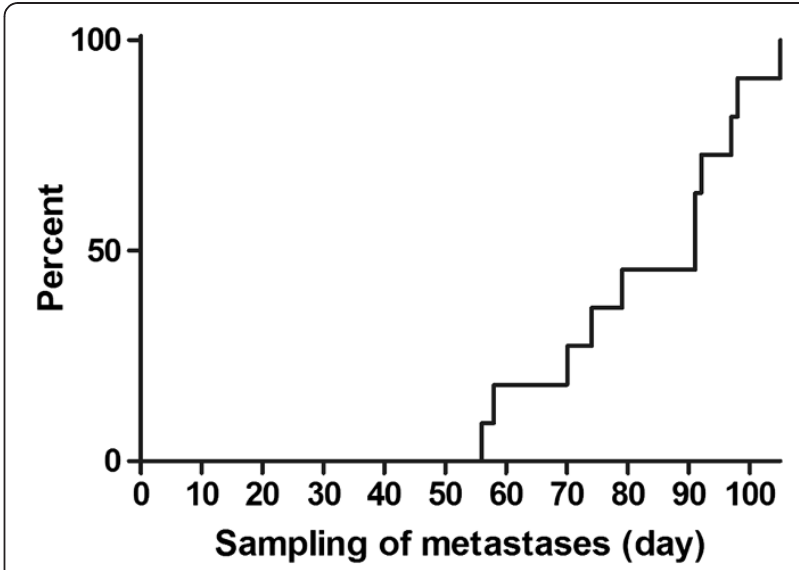

Figure 1 Timing of the detection of metastatic disease.

Accumulated percentage of rats with metastatic disease observed during the study period ( $n=11$ of 30 ). At the end of the study, two additional animals had metastases at autopsy; these are not included in this figure. Day $0=$ day of injection of ${ }^{177}$ Lu-DOTA-BR96.

BR96 target antigen and Ki67, a marker for proliferating cells. In short, $4 \mu \mathrm{m}$ thick sections were rehydrated and antigen retrieval was performed using the PT Link pretreatment module (Dako) with acidic target retrieval solution ( $\mathrm{pH} \mathrm{6)}$, preheated to $65^{\circ} \mathrm{C}$ and then heated to $99^{\circ} \mathrm{C}$ for $20 \mathrm{~min}$, followed by rinsing of the slides. The sections were incubated with BR96 or Rabbit anti Ki67 (Clone SP6, NeoMarkers, Fremont, CA) diluted in Antibody diluent (Dako) overnight (BR96) or for $2 \mathrm{~h}$ (Ki67) at room temperature. After washing, sections were incubated with anti-human IgG $\mathrm{H}+\mathrm{L}$ Donkey $\mathrm{F}(\mathrm{ab})_{2}$ HRP (Jackson ImmunoResearch Laboratories Inc., West Grove, PA) (for BR96 detection) or Donkey anti-rabbit HRP (Jackson Laboratories) (for Ki67 detection) in Antibody diluent for $3 \mathrm{~h}$ (for BR96) or $1 \mathrm{~h}$ (for Ki67) at room temperature. Finally, diaminobenzidine (Dako) was added before dehydration and mounting.

Hematoxylin and eosin staining were used to evaluate the overall tumor structure and to distinguish the tumor cells from stromal cells in the tumor. Tumor expression of the BR96 target antigen was scored. A score of 0 corresponds to less than $10 \%$ positive (strong complete membranous staining) tumor cells, 1 to $10-50 \%$ positive tumor cells, 2 to $50-90 \%$ positive tumor cells and 3 to more than $90 \%$ positive tumor cells. Proliferation was evaluated in relation to the expression of the BR96 target antigen.

Immunohistochemical staining of the BR96 target antigen was scored from 0 to 3 representing the degree of expression (see Table 1). The results presented for Group A are based on the 13 animals showing consistent CR after treatment of the primary tumor with ${ }^{177}$ Lu-DOTA-BR96 which later developed distant metastases. All the untreated primary tumors in Group B 


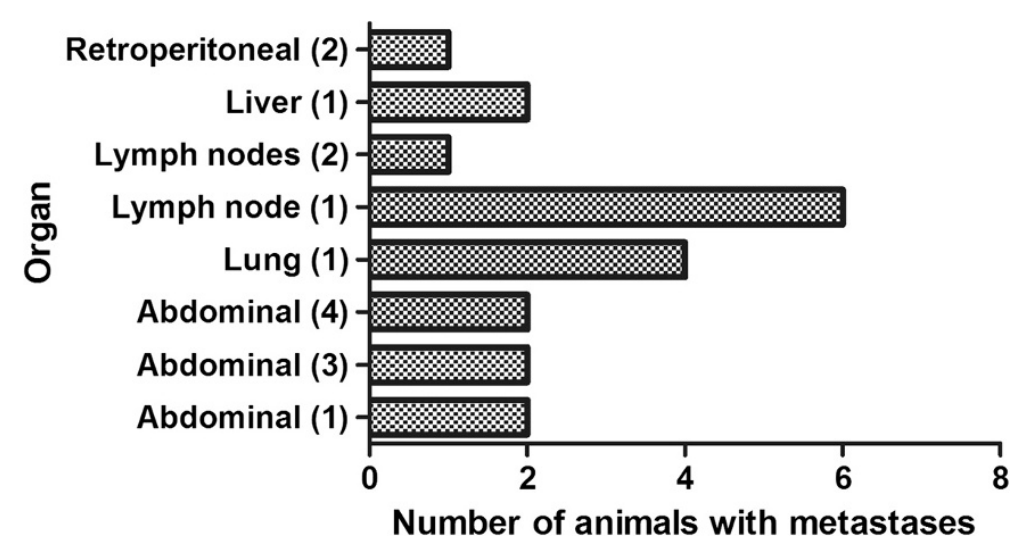

Figure 2 Tissue localization of detected metastases. The number of animals with metastases at different locations after treatment with ${ }^{177}$ Lu-DOTA-BR96. Abdominal denotes metastases growing inside the abdominal cavity but not within a specific organ, mostly in the mesentery, in fat tissue or in the peritoneum. Retroperitoneal metastases were found adjacent to the kidneys. The numbers within parentheses are the number of metastases found at the specified location in the same animal.

$(n=11)$ had a score of 3 , in contrast to the metastases in Group A, in which only 6 out of 23 had a score of 3 (Table 1). The other metastases in Group A had scores of $2(n=6), 1 \quad(n=9)$ or $0 \quad(n=2)$. The metastases with reduced antigen expression showed less BR96 binding, either in specific areas or over the whole tissue section. None of the metastases completely lacked expression of the BR96 target antigen.

Reduced proliferation was detected in 13 of 23 metastases from rats treated with radioimmunotherapy, but did not show any relation to the expression of the BR96 target antigen. All primary tumors (Group B) showed high proliferation index.

In half of the cases with more than one stained metastasis sample from the same animal, the BR96 target antigen expression score was the same. An example of different antigen expression within the same animal is illustrated in Figure 3, showing two different metastases adjacent to the right and the left kidneys. Although these two tumors had different BR96 target antigen expression (scores 2 and 1) there was no difference in proliferation.

\section{Discussion}

Primary tumors from untreated animals demonstrated an intense homogeneous BR96 target antigen staining pattern, and all were given a score of 3 . In comparison, a score of 3 was only given to 6 of the 23 distant metastases in animals belonging to Group A, showing consistent CR of the primary tumor after treatment with ${ }^{177} \mathrm{Lu}$ DOTA-BR96 (Table 1). The metastases with reduced antigen expression showed less BR96 binding, either in specific areas or over the whole tissue section. None of the metastases completely lacked expression of the BR96 target antigen. It would have been interesting to evaluate metastases in animals not administered radioimmunoconjugate, but this would have required the removal of the primary tumors to prolong the survival of these animals to allow development of metastatic disease. In untreated rats, the tumors reach maximal tolerable volume on day 21-28, i.e. prior to earliest detected metastatic disease (Figure 1). Previous attempts to surgically remove tumors inside the abdomen have not been successful. Apart from technical difficulties, surgical removal of the primary tumor might stimulate the growth of distant metastases [6]. Of the same reason we have not been able to determine whether the antigen expression of the primary tumor becomes more heterogeneous with time and local progression (exceeding $25 \times 25 \mathrm{~mm}$ ) for ethical reasons.

The antigen expression was reduced in 17 of 23 of the analyzed metastases, but it was not possible to determine whether this was due to the metastatic process itself or a result of the radioimmunotherapy, or a

Table 1 Scores reflecting the BR96 target antigen expression in metastases and primary tumors

\begin{tabular}{|c|c|c|c|c|c|c|c|}
\hline Group & Treatment & $\begin{array}{l}\text { Number of tissue samples } \\
\text { analyzed }\end{array}$ & & $\begin{array}{l}\text { Score } 0 \\
<10 \%{ }^{a}\end{array}$ & $\begin{array}{l}\text { Score } 1 \\
10-50 \%^{a}\end{array}$ & $\begin{array}{l}\text { Score } 2 \\
50-90 \%^{a}\end{array}$ & $\begin{array}{l}\text { Score } 3 \\
>90 \%{ }^{a}\end{array}$ \\
\hline $\bar{A}$ & $\begin{array}{l}{ }^{177} \text { Lu-DOTA-BR96, } \\
\text { with consistent CR }\end{array}$ & Metastases (from 13 rats) & 23 & 2 & 9 & 6 & 6 \\
\hline$B$ & No treatment & Primary tumors (from 11 rats) & 11 & 0 & 0 & 0 & 11 \\
\hline
\end{tabular}




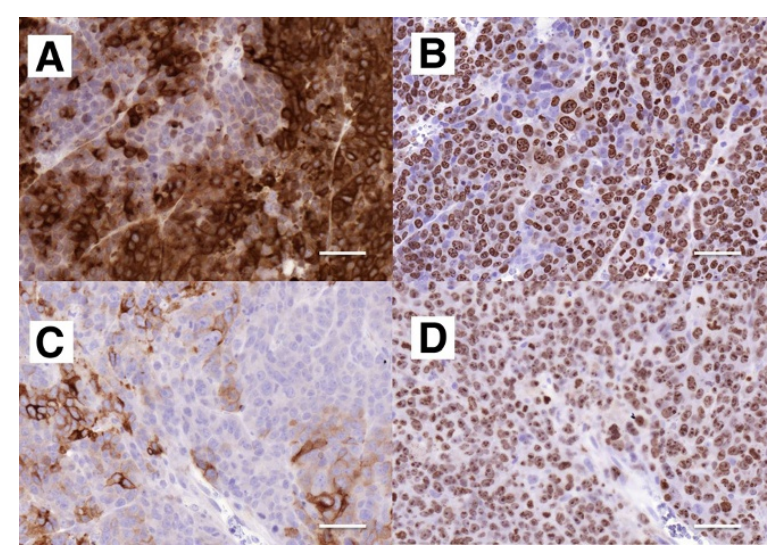

Figure 3 Different staining pattern in metastases from the same animal. Stained sections from two different metastases from the same rat treated with ${ }^{177}$ Lu-DOTA-BR96. The metastases were located retroperitoneally adjacent to the right ( $a$ and $b$ ) and left kidneys (c and d). Staining with BR96 was scored as 2 (a) and as 1 (c), and was not related to proliferation. The results for Ki67 are shown in $\mathrm{b}$ and $\mathrm{d}$. Scale bars represent $50 \mu \mathrm{m}$.

combination of both. It is not known whether tumor cells disseminate before, or as a result of, the treatment. However, no visible metastases could be detected at time of treatment.

Since none of the metastases completely lacked the BR96 target antigen, it might be beneficial to repeat radioimmunotherapy with BR96 utilizing a radionuclide with a relatively long range, resulting in irradiation from targeted to adjacent untargeted cells by the cross-fire effect. However, such radionuclides are generally regarded as unsuitable for the treatment of single cells or cell clusters. On the other hand, studies on external beam radiotherapy have indicated that it is not necessary to irradiate all the cells in a tumor to obtain efficacy as a result of the bystander effect $[7,8]$.

In experimental studies of treatment with BR96doxrubicin antibody conjugates in a rat brain tumor model, Muldoon et al. [9] reported changes in the antigen staining pattern in residual tumors. The immunoconjugate treatment resulted in outgrowth of tumors with areas of low or no antigen staining, as well as areas with moderate to intense staining, while the untreated tumors showed uniform intense staining for BR96.

Our findings indicate that during the planning of repeated radioimmunotherapy, and/or radioimmunotherapy in combination with other antibody therapies, it is important to consider the risk of reduced antigen expression in metastases, as this may result in reduced targeting of the therapeutics.

\section{Conclusions}

This study showed that the antigen expression was reduced in 17 of the 23 metastases detected after radioimmunotherapy compared with untreated tumors. Although it was not possible to demonstrate that the antigen reduction is triggered by the radioimmunotherapy this result stress the importance of considering the risk of reduced antigen expression in metastases after radioimmunotherapy prior to further targeted therapies.

\begin{abstract}
Abbreviations
BN: Brown Norway (inbred rat strain); CR: Complete response; DOTA: S-2-(4isothiocyanatobenzyl)-1,4,7,10-tetraazacyclododecane tetraacetic acid; HPLC: High performance liquid chromatography; HRP: Horseradish peroxidase; ITLC: Instant thin layer chromatography; Le': Lewis Y blood group antigen; SD: Standard deviation.
\end{abstract}

\section{Competing interests}

The authors have no affiliations, memberships, funding, or financial holdings that might be perceived as affecting the objectivity of this publication.

\section{Authors' contributions}

EE and SEE participated in the study conception and design, together carried out the animal experiments, the immunohistochemistry, the data interpretation, and drafted the manuscript. TO performed the radiochemistry. JT and RN participated in the study design and helped to draft the manuscript. All authors read and approved the final manuscript.

\section{Acknowledgments}

We would like to thank Dr. Peter Senter (Seattle Genetics, Seattle, WA) for kindly providing the monoclonal antibody BR96, Anna Ebbesson for excellent technical assistance, and Dr. Otto Ljungberg (Dept. of Pathology, Skåne University Hospital and Lund University; Sweden) for consultations regarding histology. This research was supported by grants from the Swedish Research Council, the Swedish Cancer Society, Mrs. Berta Kamprad's Foundation, Gunnar Nilsson's Foundation, The Crafoord Foundation, Government Funding of Clinical Research within the National Health Service, King Gustaf V's Jubilee Foundation, the Lund University Medical Faculty Foundation, and The Lund University Hospital Fund.

\section{Author details}

'Department of Oncology, Lund University, Lund, Sweden. ²Department of Medical Radiation Physics, Lund University, Lund, Sweden. ${ }^{3}$ Department of Oncology, Skåne University Hospital, Lund, Sweden.

Received: 24 October 2012 Accepted: 7 November 2012

Published: 13 November 2012

\section{References}

1. Sleeman J, Steeg PS: Cancer metastasis as a therapeutic target. Eur J Cancer 2010, 46:1177-1180

2. Koppe MJ, Bleichrodt RP, Oyen WJ, Boerman OC: Radioimmunotherapy and colorectal cancer. Br J Surg 2005, 92:264-276.

3. DeNardo GL, Schlom J, Buchsbaum DJ, Meredith RF, O'Donoghue JA, Sgouros G, Humm JL, DeNardo SJ: Rationales, evidence, and design considerations for fractionated radioimmunotherapy. Cancer 2002, 94:1332-1348.

4. Eriksson SE, Ohlsson T, Nilsson R, Tennvall J: Treatment with unlabeled mAb BR96 after radioimmunotherapy with 177Lu-DOTA-BR96 in a syngeneic rat colon carcinoma model. Cancer Biother Radiopharm 2012, 27:175-182.

5. Sjogren HO, Isaksson M, Willner D, Hellstrom I, Hellstrom KE, Trail PA: Antitumor activity of carcinoma-reactive BR96-doxorubicin conjugate against human carcinomas in athymic mice and rats and syngeneic rat carcinomas in immunocompetent rats. Cancer Res 1997, 57:4530-4536.

6. Coffey JC, Wang JH, Smith MJ, Bouchier-Hayes D, Cotter TG, Redmond HP: Excisional surgery for cancer cure: therapy at a cost. Lancet Oncol 2003, 4:760-768.

7. Prise KM, O'Sullivan JM: Radiation-induced bystander signalling in cancer therapy. Nat Rev Cancer 2009, 9:351-360. 
8. Formenti SC, Demaria S: Systemic effects of local radiotherapy. Lancet Oncol 2009, 10:718-726.

9. Muldoon LL, Neuwelt EA: BR96-DOX immunoconjugate targeting of chemotherapy in brain tumor models. J Neurooncol 2003, 65:49-62.

doi:10.1186/2162-3619-1-34

Cite this article as: Elgström et al:: Pattern of antigen expression in metastases after radioimmunotherapy of a syngeneic rat colon carcinoma utilizing the BR96 antibody. Experimental Hematology \& Oncology 2012 1:34

\section{Submit your next manuscript to BioMed Central} and take full advantage of:

- Convenient online submission

- Thorough peer review

- No space constraints or color figure charges

- Immediate publication on acceptance

- Inclusion in PubMed, CAS, Scopus and Google Scholar

- Research which is freely available for redistribution 